\title{
Star-shaped pill sustains drug release
}

Long-acting sustained-release oral therapies have the potential to substantially improve patient adherence and reduce side effects. However, the development of such therapeutics is limited by a rapid gastrointestinal (GI) transit time. Now, writing in Science Translational Medicine, Traverso, Langer and colleagues report the design of a gelatin-encased drug delivery system that adopts a star-shaped conformation once ingested to enable retention in the GI tract and sustained drug release while allowing safe food passage.

Although oral dosage forms that adopt a different conformation in the gastric cavity to prolong gastric

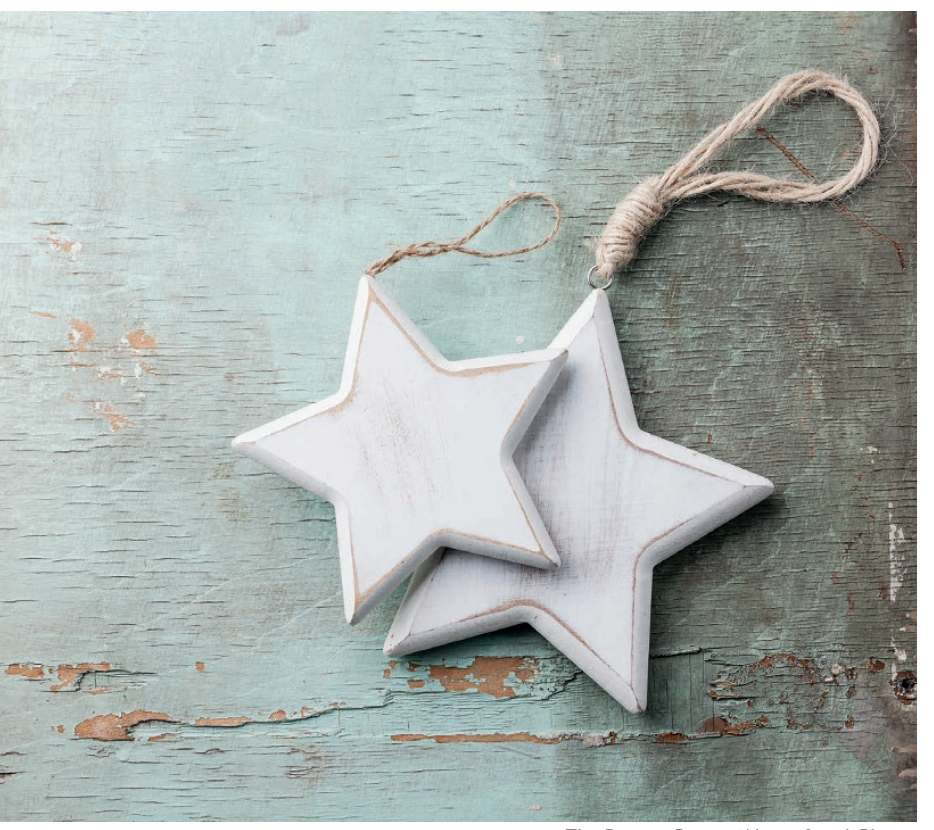

The Picture Pantry/Alamy Stock Photo residence time have previously been described, these attempts have been limited by poor safety or inadequate duration. Therefore, Traverso and colleagues set out to design an ingestible capsule that is capable of carrying large quantities of a therapeutic agent, which has the ability to safely achieve controlled sustained drug release.

Using finite element analysis and computer-aided design, the authors discovered that a stellate structure, in which six rigid arms project from a central flexible component, could be encapsulated and resist gastric contraction. They selected polycaprolactone (PCL) for the rigid drug release matrix because of its biocompatibility and established use in controlled drug delivery, whereas a thermoset polyurethane material that is composed of low-molecular-weight PCL polyols crosslinked with isocyanate was chosen as the elastic recoil element. Tunable $\mathrm{pH}$-sensitive linkers were interspersed within the PCL arms to enable control of gastric exit and intestinal dissolution, which maximizes safety in the unlikely event of the star system exiting intact into the small intestine.

Next, the authors loaded the dosage form into a soluble gelatin capsule for characterization in pigs. Abdominal X-ray analysis of more than 107 capsules, which were administered on 35 occasions to 15 different pigs, revealed that all capsules deployed properly within $5 \mathrm{~min}$, allowing the 6 arms to unfold into a star-like structure within the gastric cavity, which remained there for up to 10 days. Importantly, even after prolonged periods of gastric residence, there was no evidence of muscosal injury, erosions or ulceration, or signs of GI obstruction.

Next, they suspended ivermectin (which targets malaria-transmitting mosquitoes) into the PCL matrix of the dosage form and endoscopically administered the capsule-enclosed dosage forms to pigs. Sustained serum levels of ivermectin within a target therapeutic range for the reduction of malaria transmission was achieved for more than 10 days without evidence of GI obstruction or mucosal injury.

Finally, using mathematical modelling of malaria transmission, the authors predicted that sustaining ivermectin at mosquitocidal levels would potentiate the efficacy of a mass drug treatment based on artemisinin combination therapy for malaria and increase the probability of achieving elimination.

This novel delivery platform has the potential to be used with any orally available drug, replace frequent dosing schedules with a single administration and revolutionize treatment options for malaria and other diseases that affect large populations.

Sarah Crunkhorn

ORIGINAL ARTICLE Bellinger, A. M. et al. Oral, ultra-long-lasting drug delivery: application toward malaria elimination goals. Sci. Transl Med. 8,365ra157 (2016) 